\title{
Preparation of Hollow Titania Nanoparticles of Single-nanometer Size Using a PAMAM Dendrimer Template
}

\author{
Nao Sorida ${ }^{1}$, Shuichiro Hakoda ${ }^{1}$, Kanjiro Torigoe ${ }^{1 *}$, Takeshi Endo ${ }^{1}$, Kenichi Sakai $^{2}$, \\ Hideki Sakai ${ }^{1,2}$ and Masahiko Abe ${ }^{1,2}$ \\ ${ }^{1}$ Department of Pure and Applied Chemistry, Tokyo University of Science, 2641 Yamazaki, Noda 278-8510 Japan \\ ${ }^{2}$ Research Institute for Science and Technology, Tokyo University of Science, 2641 Yamazaki, Noda 278-8510 Japan
}

\begin{abstract}
Hollow titania nanoparticles of single-nanometer size have been prepared from titanium oxysulfate ( $\mathrm{TiOSO}_{4}$ ) using poly(amidoamine) (PAMAM) dendrimer molecules (Generations 5 with amino terminal groups) as a template. At low $\mathrm{pH}(\mathrm{pH} 1)$, hydrolysis of the titania precursor was attenuated and the sol-gel reaction preferentially proceeded at the dendrimer surface. Calcination at $450^{\circ} \mathrm{C}$ yielded crystalline titania nanoparticles of $5.5 \mathrm{~nm}$ diameter with a $\sim 2 \mathrm{~nm}$ cavity. These hollow titania nanoparticles showed a larger band-gap energy than solid particles of corresponding size.
\end{abstract}

Key words: titania, hollow nanoparticles, PAMAM, dendrimer, template

\section{INTRODUCTION}

Titanium dioxide or titania is one of the most extensively explored semiconductor materials for various applications such as pigments ${ }^{1)}$, optical coatings ${ }^{2,3)}$, wettability control ${ }^{4,5)}$, photocatalysis $^{6-8)}$ and photovoltaics ${ }^{9,10)}$. In particular, hollow titania nanoparticles have attracted increasing attention due to their lower density and higher reflectivity compared with the solid ones. Several different approaches have been developed for their synthesis, which can be classified into template-assisted ${ }^{11-13)}$ and template-free methods ${ }^{14-19)}$. In the former method, hard (e.g., polymer latex) or soft templates (microemulsions, vesicles) are utilized. Template-assisted methods can provide hollow nanoparticles with uniform sizes, but the removal of templates by dissolution or calcination process often causes disruption of particles. In the latter method, hollow titania nanoparticles are produced by Ostwald ripening ${ }^{14)}$ or chemically or thermally induced self-transformation of solid particles ${ }^{15-17)}$. These template-free methods are free from the removal problems, but size uniformity is attainable for limited systems and the crystallization of titania while maintaining hollow structure is not straightforward. Furthermore, in both methods, the hollow titania nanoparticles have diameters of more than several tens of nanometers and the fabrication of smaller hollow particles is still challenging.
It is well known that the band gap energy of titania depends on the particle size $\mathrm{e}^{20-22)}$. Moreover, a recent theoretical study ${ }^{23)}$ indicated that various properties of titania, e.g., sun protection factor (SPF), transparency in the visible regime, and production of reactive oxygen species (ROS), are also dependent on the nanoparticle size. Interestingly, SPF and the visible transparency are maximal for the diameter of $25 \mathrm{~nm}$ or less, while the production of ROS shows the maximum at about $35 \mathrm{~nm}$ and steeply decreases with further decrease in the particle size ${ }^{23)}$. Accordingly, the synthesis of titania nanoparticles with single-nanometer size is significant not only in basic research but also in practical applications.

We report herein the synthesis of monodisperse hollow titania nanoparticles of about $5 \mathrm{~nm}$ in diameter using a dendrimer as a template. Dendrimers have several unique characteristics, i.e. uniform molecular size and chemically modifiable terminal groups ${ }^{24)}$. Regarding the dendrimer-assisted synthesis of titania nanoparticles, Imae and co-work$\mathrm{ers}^{25,26)}$ reported poly (amidoamine) (PAMAM) dendrimersprotected titania nanoparticles using $\mathrm{TiCl}_{4}$ as a precursor, as well as their photocatalytic properties for the photodegradation of an organic pollutant. Each titania nanoparticle was protected with several dendrimer molecules, and thus, the role of dendrimers was not being a template but a stabilizer for titania nanoparticles. On the other hand, Kunita-

\footnotetext{
*Correspondence to: Kanjiro Torigoe, Department of Pure and Applied Chemistry, Tokyo University of Science, 2641 Yamazaki, Noda 278-8510 Japan

E-mail: ktorigoe@rs.noda.tus.ac.jp

Accepted March 13, 2013 (received for review February 18, 2013)

Journal of Oleo Science ISSN 1345-8957 print / ISSN 1347-3352 online

http://www.jstage.jst.go.jp/browse/jos/ http://mc.manusriptcentral.com/jjocs
} 
ke and co-workers ${ }^{27)}$ prepared mesoporous titania film using glass-supported PAMAM dendrimers as a template for mesopores. More recently Yamamoto and co-workers ${ }^{28)}$ reported the quantum size effect of small titania clusters prepared by the coordination of $\mathrm{Ti}$ (acac) $\mathrm{Cl}_{3}$ with poly (azomethyne) dendrimers.

We report herein that individual PAMAM dendrimer molecules can act as a template for titania nanoparticles and that the removal of dendrimer templates by calcinations leads to monodispersed hollow nanoparticles.

\section{EXPERIMENTAL}

Fifth generation poly (amidoamine) dendrimers (denoted as PAMAM G5) with 128 terminal amino groups per molecule was purchased as $5 \mathrm{wt} \%$ solution in methanol from Aldrich and used as received. After removing the solvent with a rotary evaporator, $5.56 \times 10^{-5} \mathrm{~mol}$ (terminal group concentration) of dendrimer was dissolved in $5 \mathrm{~cm}^{3}$ of deionized water (Barnstead Nanopure). After stirring for 30 min at ambient temperature, $\mathrm{pH}$ was adjusted with sulfuric acid (initial $\mathrm{pH}$ was 10). Separately, $0.69 \mathrm{~g}$ of titanium(IV) oxysulfate $\left(\mathrm{TiOSO}_{4}\right.$, Aldrich) was dissolved in $100 \mathrm{~cm}^{3}$ of water to make $0.5 \mathrm{~mol} \mathrm{dm}^{-3}$ aqueous solution of the titania precursor. Subsequently a $100 \mathrm{~mm}^{3}$ aliquot of the precursor solution was quickly injected into the dendrimer solution under vigorous stirring. The mixture was left stirring for $1 \mathrm{~d}$ at ambient temperature. Then, the whole amount of aqueous titania dispersion was transferred to an alumina crucible and calcined at $450^{\circ} \mathrm{C}$ for $2 \mathrm{~h}$ with heating rate at $1^{\circ} \mathrm{C} \mathrm{min}^{-1}$. Ten milligrams of the powder product was redispersed in $5 \mathrm{~cm}^{3}$ of water. For TEM analysis, $2 \mathrm{~mm}^{3}$ of the supernatant was mounted on carbon-coated $\mathrm{Cu}$ grids and imaged with Hitachi H-7650 (120 kV) or H-9500 (200 $\mathrm{kV}$ ) transmission electron microscope. Energy-dispersive X-ray analysis was performed with a Horiba EDX system attached with H-7650. UV-vis absorption spectra were re- corded on an Agilent 8453 diode array spectrophotometer at $1 \mathrm{~cm}$ of light path.

\section{RESULTS AND DISCUSSION}

Figure 1 presents the TEM images of titania particles prepared at different $\mathrm{pH}$ conditions. Well-dispersed spherical nanoparticles of $15 \mathrm{~nm}$ in diameter are found at $\mathrm{pH} 1$. Closer observation reveals that each particle contains a 6-nm pore, which is close to the molecular size of PAMAM $\mathrm{G} 5(6.6 \mathrm{~nm})^{24)}$. With increasing $\mathrm{pH}$, large and irregularshaped particles become abundant. This is because at $\mathrm{pH} 1$, the hydrolysis of $\mathrm{TiOSO}_{4}$ is slow and proceeds mainly on the amino groups at the dendrimer surface, whereas at higher $\mathrm{pH}$, rapid hydrolysis proceeds at bulk solution phase. Accordingly, all experiments described hereafter were performed at $\mathrm{pH} 1$.

Figure S1 presents the EDX profile of as-prepared titania nanoparticles at $\mathrm{pH} 1$. Three discernible peaks are detected for Ti corresponding to $L_{\alpha}(0.452$ and $0.458 \mathrm{keV})$, $K_{\alpha}(4.508 \mathrm{keV})$ and $K_{\beta 1}(4.931 \mathrm{keV})$ lines, and one peak for O corresponding to $\left.K_{\alpha}(0.525 \mathrm{keV}) \operatorname{line}^{29}\right)$. Apart from these two elements, an S $K_{\alpha}$ line $(2.307 \mathrm{keV})$ was detected, originating from sulfate ions adsorbed on the titania surface. Meanwhile, the electron diffraction presents a hallow pattern(not shown), indicating the amorphous nature of these nascent titania nanoparticles.

Successively, this sample was calcined at $450^{\circ} \mathrm{C}$ for $2 \mathrm{~h}$. When the powder was redispersed in water, most particles aggregated and precipitated. However, a small fraction (ca. $5 \mathrm{wt} \%$ ) could be dispersed in the supernatant, where monodisperse titania nanoparticles were observed. When PAMAM G5 was used as a template, the mean particle size was $5.5 \mathrm{~nm}(\sigma=0.76 \mathrm{~nm})$, as shown in Fig. 2a. Compared with the as-prepared sample, an obvious size decrease was detected. In addition, the magnified images (Fig. $2 \mathrm{~b}$ and Fig. S2)indicate a $\sim 2$ nm cavity for each particle. Appar- (a) $\mathrm{pH} 1$

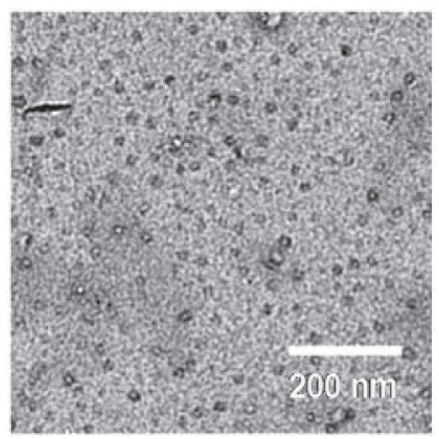

(b) $\mathrm{pH} 2$

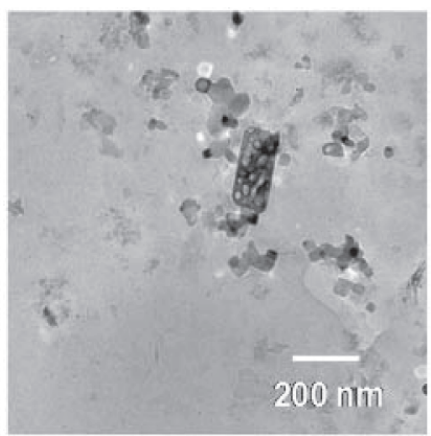

(c) $\mathrm{pH} 3$

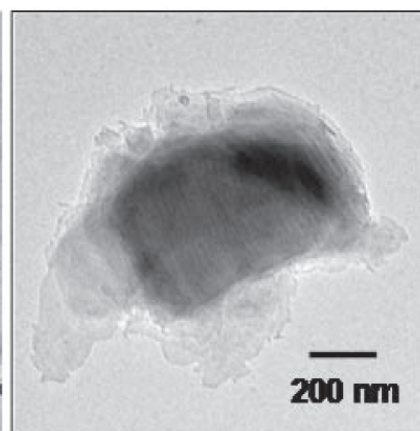

(d) $\mathrm{pH} 6$

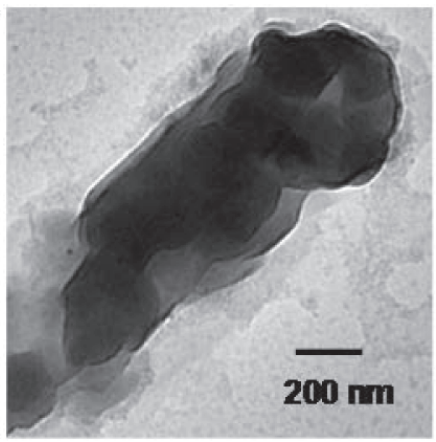

Fig. 1 TEM images of titania nanoparticles prepared from the hydrolysis of $\mathrm{TiOSO}_{4}$ in the presence of PAMAM G5 at various $\mathrm{pH}$ conditions. 


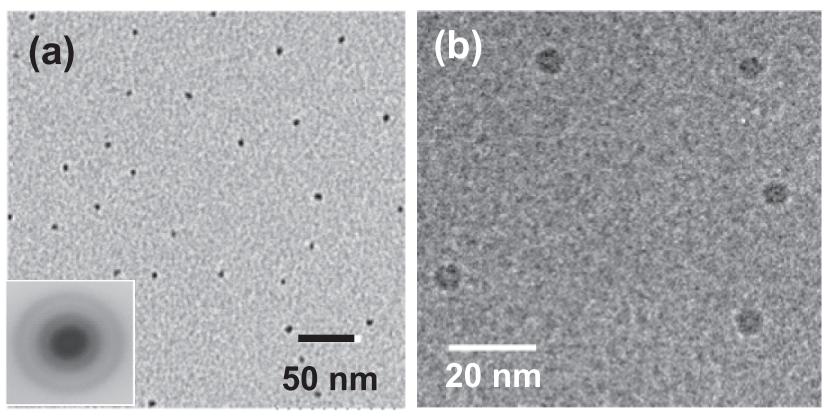

Fig. 2 (a) TEM image of titania nanoparticles formed with PAMAM G5 and (inset) their electron diffraction pattern, (b) high-magnification image of (a).

ently burning off of the dendrimer template and crystallization of titania nanoparticles are taking place during the calcination process. When we change the template from PAMAM G5 to G7, there was a slight increase in the particle $\operatorname{size}(d=6.2 \mathrm{~nm} ; \sigma=0.67 \mathrm{~nm})$. This is because the size decrease due to burning off of the template and crystallization of titania counterbalances the increased size of template molecules with higher generations.

The crystalline nature of calcined titania nanoparticles can be verified from Debye-Scherrer rings of the electron diffraction pattern (Fig. 2a inset). However, it is difficult to determine unambiguously whether these particles are anatase, rutile, or their mixture, because of the significant broadening of diffraction rings associated with small particle size. Note that these nanometer-sized titania nanoparticles were obtained only when ionic titanium complex (titanium oxysulfate) was employed as a precursor, although we tried nonionic titanium complexes, e.g., titanium tetraisopropoxide and titanium tetrabutoxide. This suggests that electrostatic interaction between the titania precursor and dendrimer surface plays a crucial role in the formation of hollow titania particles.

As we previously reported for the preparation of mesoporous titania in the presence of cetyltrimethylammonium bromide $(\mathrm{CTAB})^{30)}$, quaternary ammonium group promotes the hydrolysis and condensation of $\mathrm{TiOSO}_{4}$. We found that micellization of $\mathrm{C}_{n} \mathrm{TAB}(\mathrm{n}=$ alkyl chain length $)$ is crucially important for a template, since non-micelle-forming $\mathrm{C}_{\mathrm{n}} \mathrm{TAB}$ bearing shorter alkyl chains $(\mathrm{n} \leq 8)$ do not function as a template. Similarly, when we tried PAMAM dendrimers of different generations from G3 to G7, only generations higher than G5 proved to work as templates. This suggests that the congestion of surface amino groups is a prerequisite for the formation of hollow titania nanoparticles.

Figure 3 displays a UV-vis extinction spectrum of hollow titania nanoparticles derived from PAMAM G5 template. The threshold wavelength (energy) is $340 \mathrm{~nm}(3.65 \mathrm{eV})$, which is significantly blue-shifted from that of bulk titania in anatase form, $380 \mathrm{~nm}(3.26 \mathrm{eV})$. Moreover, the band-gap

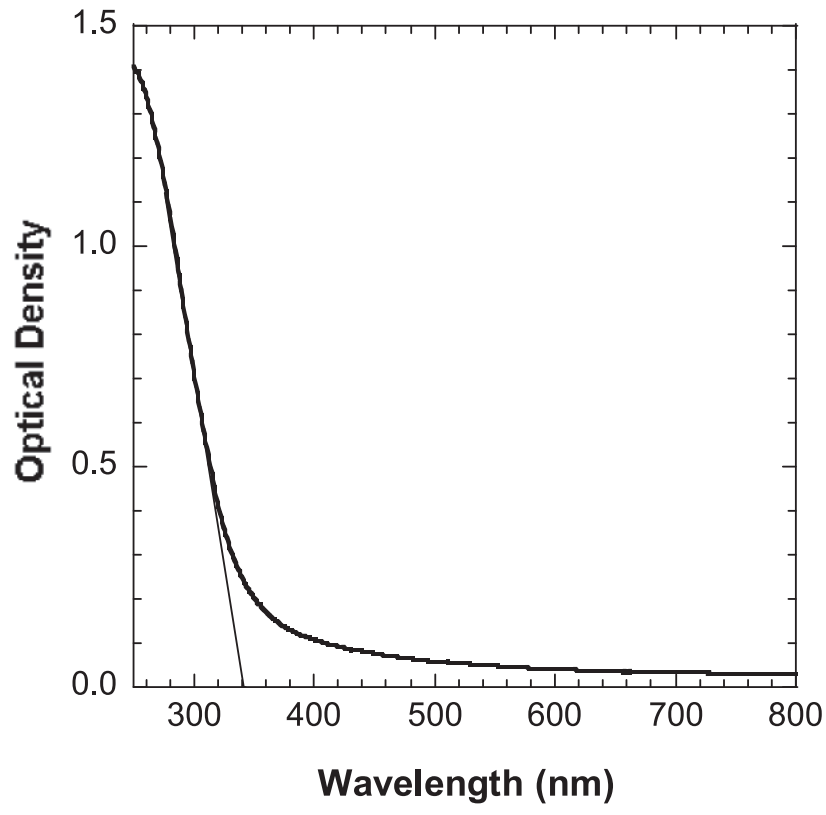

Fig. 3 UV-vis extinction spectrum of hollow titania nanoparticles in aqueous dispersion.

wavelength of these hollow particles is significantly blueshifted from that of solid nanoparticles of corresponding size $(375 \mathrm{~nm})^{20)}$. This can be attributed to thin walls of hollow titania nanoparticles.

\section{CONCLUSION}

Hollow titania nanoparticles of single-nanometer size $(d$ $\sim 5.5 \mathrm{~nm}$, pore size $\sim 2 \mathrm{~nm}$ ) can be prepared from $\mathrm{TiOSO}_{4}$ using PAMAM G5 molecules as a template. Attenuated hydrolysis of titania precursor at strongly acidic condition $(\mathrm{pH}$ 1 ) is essential. Crystalline nature of calcined titania nanoparticles was confirmed by electron diffraction. Electrostatic interaction between the titania precursor and the amino groups at the dendrimer surface is responsible for the success of dendrimer molecules as a template. The threshold wavelength $(340 \mathrm{~nm}$ ) of hollow nanoparticles was significantly blue-shifted from those of bulk titania (anatase) and solid anatase particles of corresponding size.

\section{Supporting Information}

This material is available free of charge via the Internet at http://dx.doi.org/10.5650/jos.62.637

\section{ACKNOWLEDGMENT}

We are grateful for Prof. Iwao Hashimoto (TUS) and Prof. 
Makoto Tadokoro(TUS) for providing us their TEM facilities (H-9500).

\section{REFERENCES}

1) Balfour, J. G. Titanium dioxide pigments in Technological applications of dispersions (McKay, R. B. ed.) Surfactant Science Series, Vol. 52, Marcel Dekker, pp. 69-104 (1994).

2) Hao, H.; Li, B. Photothermal detuning for absorption measurement of optical coatings, Appl. Opt. 47, 188194 (2008).

3) Hidalgo, N.; Calvo, M. E.; Miguez, H. Mesostructured thin films as responsive optical coatings of photonic crystals, Small 5, 2309-2315 (2009).

4) Kanta, A.; Sedev, R.; Ralston, J. Preparation of silicaon-titania patterns with a wettability contrast, Langmuir 21, 5790-5794 (2005).

5) Zhang, M.; Zhang, T.; Cui, T. Wettability conversion from superoleophobic to superhydrophilic on titania / single-walled carbon nanotube composite coatings, Langmuir 27, 9295-9301 (2011).

6) Anpo, M.; Kamat, P. Environmentally benign photocatalysis: Applications of titanium oxide-based materials, Wiley, 2011.

7) Kumar, S. G.; Devi, L. G. R. Review on modified $\mathrm{TiO}_{2}$ photocatalysis under UV/visible light: selected results and related mechanisms on interfacial charge carrier transfer dynamics, J. Phys. Chem. A 115, 1321113241 (2011).

8) Li, Y.-F.; Liu, Z.-P. Particle size, shape and activity for photocatalysis on titania anatase nanoparticles in aqueous surroundings, J. Am. Chem. Soc. 133, 1574315752 (2011).

9) Franco, G.; Gehring, J.; Peter, L. M.; Ponomarev, E. A.; Uhlendorf, I. Frequency-resolved optical detection of photoinjected electrons in dye-sensitized nanocrystalline photovoltaic cells, J. Phys. Chem. B 103, 692-698 (1999).

10) O’Regan, B. C.; Lenzmann, F. Charge transport and recombination in a nanoscale interpenetrating network of n-type and p-type semiconductors: Transient photocurrent and photovoltage studies of $\mathrm{TiO}_{2} / \mathrm{Dye} / \mathrm{CuS}-$ CN photovoltaic cells, J. Phys. Chem. B 108, 43424350 (2004).

11) Caruso, R. A.; Susha, A.; Caruso, F. Multilayered titania, silica, and laponite nanoparticle coatings on polystyrene colloidal templates and resulting inorganic hollow spheres, Chem. Mater. 13, 400-409 (2001).

12) Song, C.; Yu, W.; Zhao, B.; Zhang, H.; Tang, C.; Sun, K.; Wu, X.; Dong, L.; Chen, Y. Efficient fabrication and photocatalytic properties of $\mathrm{TiO}_{2}$ hollow spheres, Catal. Commun. 10, 650-654(2009).
13) Yoon, S.; Manthiram, A. Hollow core-shell mesoporous $\mathrm{TiO}_{2}$ spheres for lithium ion storage, J. Phys. Chem. C 115, 9410-9416 (2011).

14) Yang, H. G.; Zeng, H. C. Preparation of hollow anatase $\mathrm{TiO}_{2}$ nanospheres via Ostwald ripening, J. Phys. Chem. B 108, 3492-3495 (2004).

15) Yu, J.; Guo, H.; Davis, S. A.; Mann, S. Fabrication of hollow inorganic microspheres by chemically induced self-transformation, Adv. Funct. Mater. 16, 2035-2041 (2006).

16) Li, H.; Bian, Z.; Zhu, J.; Zhang, D.; Li, G.; Huo, Y.; Li, H.; $\mathrm{Lu}, \mathrm{Y}$. Mesoporous titania spheres with tunable chamber structure and enhanced photocatalytic activity, $J$. Am. Chem. Soc. 129, 8406-8407(2007).

17) Kim, Y. J.; Chai, S. Y.; Lee, W. I. Control of $\mathrm{TiO}_{2}$ structures from robust hollow microspheres to highly dispersible nanoparticles in a tetrabutylammonium hydroxide solution, Langmuir 23, 9567-9571 (2007).

18) Lü, X.; Ding, S.; Xie, Y.; Huang, F. Non-aqueous preparation of high-crystallinity hierarchical $\mathrm{TiO}_{2}$ hollow spheres with excellent photocatalytic efficiency, Eur. J. Inorg. Chem. 2011, 2879-2883 (2011).

19) Shang, S.; Jiao, X.; Chen, D. Template-free fabrication of $\mathrm{TiO}_{2}$ hollow spheres and their photocatalytic properties, ACS Appl. Mater. Interface 4, 860-865 (2012).

20) Anpo, M.; Shima, T.; Kodama, S.; Kubokawa, Y. Photocatalytic hydrogenation of propyne with water on small-particle titania: size quantization effects and reaction intermediates, J. Phys. Chem. 91, 4305-4310 (1987).

21) Kormann, C.; Bahnemann, D. W.; Hoffmann, M. R. Preparation and characterization of quantum-size titanium dioxide, J. Phys. Chem. 92, 5196-5201(1988).

22) Serpone, N.; Lawless, D.; Khairutdinov, R. Size effects on the photophysical properties of colloidal anatase $\mathrm{TiO}_{2}$ particles: Size quantization versus direct transitions in this indirect semiconductor? J. Phys. Chem. 99, 16646-16654 (1995).

23) Barnard, A. S. One-to-one comparison of sunscreen efficacy, aesthetics and potential nanotoxicity, Nature Nanotech. 5, 271-274(2010).

24) Tomalia, D. A.; Naylor, A. M.; Goddard III, W. A. Starburst dendrimers: Molecular-level control of size, shape, surface chemistry, topology, and flexibility from atoms to macroscopic matter, Angew. Chem. Int. Ed. Engl. 29, 138-175 (1990).

25) Nakanishi, Y.; Imae, T. Synthesis of dendrimer-protected $\mathrm{TiO}_{2}$ nanoparticles and photodegradation of organic molecules in an aqueous nanoparticle suspension, $J$. Colloid Interface Sci. 285, 158-162 (2005).

26) Nakanishi, Y.; Imae, T. Preparation of siloxy focal dendron-protected $\mathrm{TiO}_{2}$ nanoparticles and their photocatalysis, J. Colloid Interface Sci. 297, 122-129 (2006).

27) Huang, J.; Ichinose, I.; Kunitake, T. Replication of den- 
drimer monolayer as nanopores in titania ultrathin film, Chem. Commun. 2070-2071 (2002).

28) Saitoh, N.; Nakashima, T.; Kamimura, K.; Yamamoto, K. Quantum size effect in $\mathrm{TiO}_{2}$ nanoparticles prepared by finely controlled metal assembly on dendrimer templates, Nat. Nanotech. 3, 106-111 (2008).
29) CRC handbook of chemistry and physics, $81^{\text {st }}$ Ed. CRC Press, 2001.

30) Shibata, H.; Ogura, T.; Mukai, T.; Ohkubo, T.; Sakai, H.; Abe, M. Direct synthesis of mesoporous titania particles having a crystalline wall, J. Am. Chem. Soc. 127, 16396-16397(2005). 